This item was submitted to Loughborough's Research Repository by the author.

Items in Figshare are protected by copyright, with all rights reserved, unless otherwise indicated.

\title{
Radiating solitary waves in coupled Boussinesq equations
}

PLEASE CITE THE PUBLISHED VERSION

https://doi.org/10.1093/imamat/hxx014

\section{PUBLISHER}

(c) the Authors. Published by Oxford University Press

\section{VERSION}

AM (Accepted Manuscript)

\section{PUBLISHER STATEMENT}

This work is made available according to the conditions of the Creative Commons Attribution-NonCommercialNoDerivatives 4.0 International (CC BY-NC-ND 4.0) licence. Full details of this licence are available at: https://creativecommons.org/licenses/by-nc-nd/4.0/

\section{LICENCE}

CC BY-NC-ND 4.0

\section{REPOSITORY RECORD}

Grimshaw, Roger H.J., Karima R. Khusnutdinova, and Kieron R. Moore. 2019. "Radiating Solitary Waves in Coupled Boussinesq Equations". figshare. https://hdl.handle.net/2134/24858. 


\title{
Radiating solitary waves in coupled Boussinesq equations
}

\author{
R.H.J. Grimshaw, K.R. Khusnutdinova and K.R. Moore \\ Department of Mathematical Sciences, Loughborough University, \\ Loughborough LE11 3TU, UK
}

Corresponding author: K.Khusnutdinova@lboro.ac.uk

\begin{abstract}
In this paper we are concerned with the analytical description of radiating solitary wave solutions of coupled regularised Boussinesq equations. This type of solution consists of a leading solitary wave with a small-amplitude co-propagating oscillatory tail, and emerges from a pure solitary wave solution of a symmetric reduction of the full system. We construct an asymptotic solution, where the leading order approximation in both components is obtained as a particular solution of the regularised Boussinesq equations in the symmetric case. At the next order, the system uncouples into two linear non-homogeneous ordinary differential equations with variable coefficients, one correcting the localised part of the solution, which we find analytically, and the other describing the co-propagating oscillatory tail. This latter equation is a fourth order ordinary differential equation and is solved approximately by two different methods, each exploiting the assumption that the leading solitary wave has a small amplitude, and thus enabling an explicit estimate for the amplitude of the oscillating tail. These estimates are compared with corresponding numerical simulations.
\end{abstract}

Keywords: Coupled Boussinesq equations; Radiating solitary waves; Perturbation theory. 


\section{Introduction}

In the last couple of decades it has been shown that certain Boussinesq-type equations can be used to model the propagation of long nonlinear longitudinal bulk strain waves in many elastic waveguides, including for example rods and plates, see Samsonov (2001); Porubov (2003). The predicted longitudinal bulk strain solitary waves were observed in experiments by Dreiden et al (1988); Samsonov et al (1998); Semenova et al (2005). Recently, these theoretical and experimental studies were extended to some types of adhesively bonded layered bars by Khusnutdinova and Samsonov (2008); Khusnutdinova et al (2009); Dreiden et al (2011, 2012). In particular, it was shown that nonlinear longitudinal bulk strain waves in a by-layer with a sufficiently soft adhesive bonding can be modelled by a system of coupled regularised Boussinesq (cRB) equations:

$$
\begin{aligned}
f_{t t}-f_{x x} & =\frac{1}{2}\left(f^{2}\right)_{x x}+f_{t t x x}-\delta(f-g), \\
g_{t t}-c^{2} g_{x x} & =\frac{1}{2} \alpha\left(g^{2}\right)_{x x}+\beta g_{t t x x}+\gamma(f-g) .
\end{aligned}
$$

The equations are written in non-dimensional form, $f$ and $g$ describe the longitudinal strains in the layers, while the coefficients are defined by the physical and geometrical parameters of the problem (see Khusnutdinova et al (2009) for details).

In the symmetric case, when $c=\alpha=\beta=1$, system (1) admits a reduction $g=f$, where $f$ satisfies the single regularised Boussinesq equation

$$
f_{t t}-f_{x x}=\frac{1}{2}\left(f^{2}\right)_{x x}+f_{t t x x} .
$$

The Boussinesq equation (2) has particular solutions in the form of pure solitary waves:

$$
f=a \operatorname{sech}^{2} \frac{x-v t}{\Lambda}, \quad a=3\left(v^{2}-1\right), \quad \Lambda=\frac{2 v}{\sqrt{v^{2}-1}} .
$$

Figure 1 illustrates the pure solitary wave solution (3) for two values of the parameter $v$.

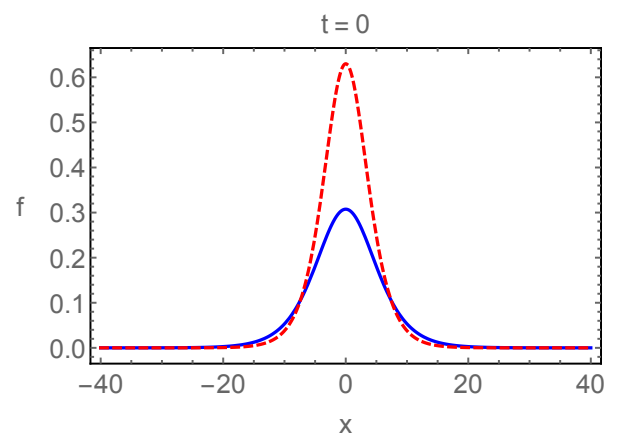

Figure 1: Pure solitary wave solution (3) for $v=1.05$ (solid line) and $v=1.1$ (dashed line).

However, in the cRB system of equations (1), when the value of $c$ is close to 1 , that is, the characteristic speeds of the linear waves in the layers are close, these "pure" or "classical" solitary wave solutions, rapidly decaying to zero in their tail regions, are structurally unstable and are replaced with "radiating" solitary waves (see Khusnutdinova et al (2009); Khusnutdinova and Moore (2011)), that is a solitary wave radiating a co-propagating one-sided oscillatory tail, using the terminology in Benilov et al (1993); Voronovich et al (2006); Bona et al (2008). A typical illustration of radiating solitary wave solutions in each component of the cRB equations (1) is depicted in Figure 2. 

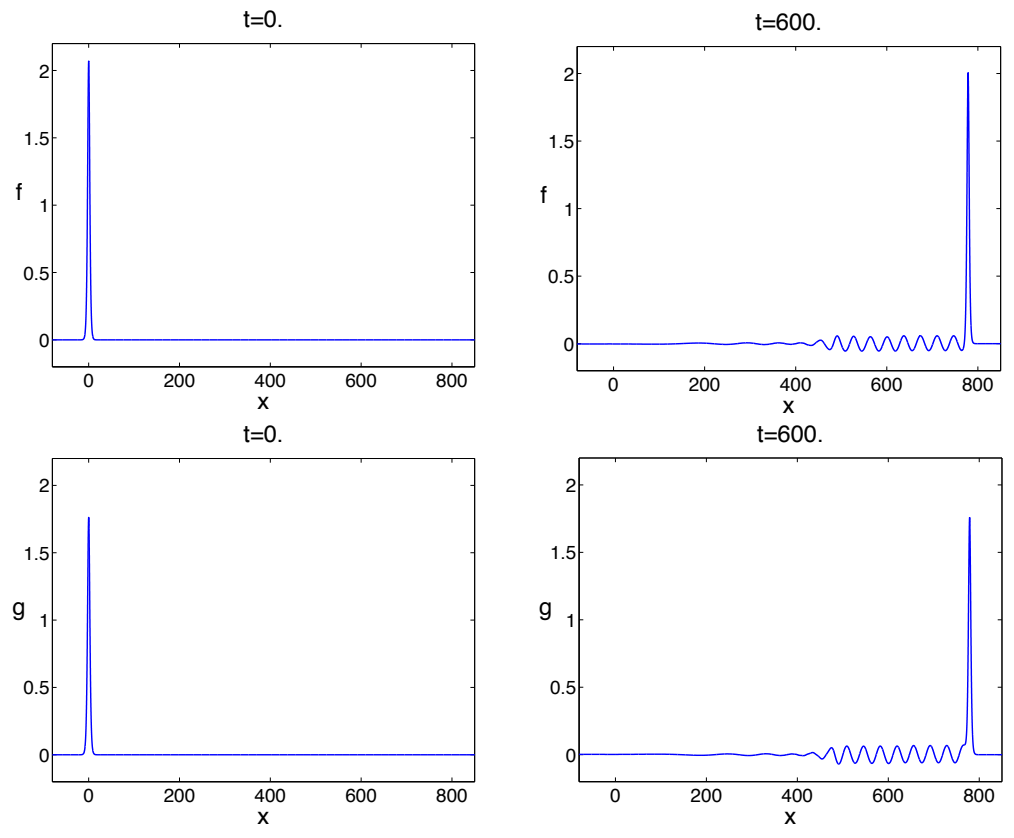

Figure 2: Typical generation of radiating solitary waves in the system of cRB equations (1), from pure solitary wave initial conditions. Here $c=1.05, \alpha=\beta=1, \gamma=\delta=0.01 ; v=1.3$.

There have been extensive studies of generalised and radiating solitary waves, especially in the context of fluid mechanics, see for instance, Boyd (1998); Fochesato et al (2005); Grimshaw and Iooss (2003); Grimshaw and Joshi (1995); Karpman (1993); Lombardi (2000); Vanden-Broeck (1991)). The most commonly studied systems supporting these non-local nonlinear long waves include perturbed KdV equations; coupled KdV systems; perturbed NLS equations and coupled NLS systems. The underlying reason for the occurrence of generalised and radiating solitary waves is due to a resonance between a long wave (with wavenumber $k \approx 0$ ) and a short wave (with some finite wavenumber). Steady generalised solitary waves are necessarily symmetric, and they support oscillating tails on both sides of the localised central core. However this means they usually cannot be realised physically since the group velocity of the oscillating tails is the same at both ends. In practice, one instead finds that radiating solitary waves are generated, that is asymmetric non-local solitary waves with the oscillating tail appearing on only that side of the central core determined by the outgoing group velocity. Strictly, such radiating solitary waves must be unsteady, and decay due to the emitted radiation. But if the amplitude of this radiating tail is sufficiently small, then this rate of decay is sufficiently small that a quasi-steady assumption can be made.

In the system (1) considered here, long-wave oscillations are radiated from the central localised core, due to the nature of the coupling terms in the equations and the resulting structure of the linear dispersion relation. The linear dispersion relation for (1) was analysed in Khusnutdinova et al (2009) by assuming that the coefficients in (1) are perturbed compared to the symmetric case, but remain positive. The dispersion relation has the form

$$
\left[k^{2}\left(1-p^{2}\right)-k^{4} p^{2}+\delta\right]\left[k^{2}\left(c^{2}-p^{2}\right)-\beta k^{4} p^{2}+\gamma\right]=\gamma \delta,
$$

where $k$ is the wavenumber and $p$ is the phase speed. A typical plot of (4) is shown in Figure 3(a). A significant difference with the linear dispersion curve of the reduction (2) consists in the appearance of the second (upper) branch, going to infinity as $k \rightarrow 0$, and approaching zero, remaining above the lower branch, as $k \rightarrow \infty$. 


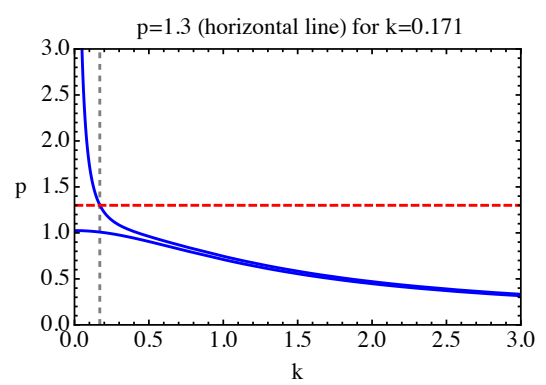

(a)

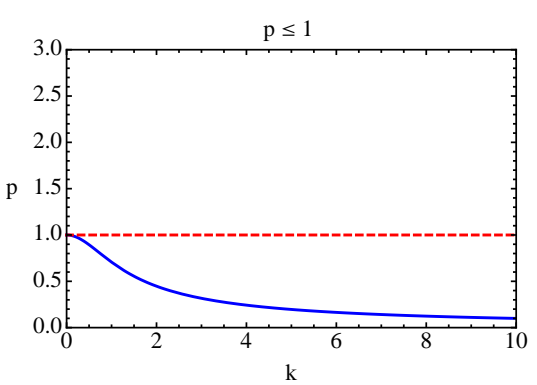

(b)

Figure 3: (a) Two branches of the linear dispersion curve of the cRB equations (1) for $c=1.05, \beta=1, \delta=\gamma=0.01$ and illustration of a possible resonance for $p=1.3$ (horizontal line), and (b) Linear dispersion curve of the reduction (2) in the symmetric case $c=1, \beta=1, \delta=\gamma=0.01$.

The classical or pure solitary waves of the single Boussinesq equation (2) arise as a bifurcation from wavenumber $k=0$ of the linear wave spectrum, shown in Figure 3(b), when there is no possible resonance with any linear wave for any value of $k$. The solitary wave speed $v$ is greater than the linear long wave speed, that is, $v>1$, while the speed of a linear wave of any wavenumber is smaller, that is $p \leq 1$. This becomes impossible when the symmetry is broken. Instead, radiating solitary waves arise for the case when there is a possible resonance with the upper branch for some finite non-zero value of $k$. For example, a possible resonance is shown in Figure 3(a) for $v=p=1.3$. Recently, radiating solitary waves have been experimentally observed in two- and three-layered elastic waveguides with soft bonding layers by Dreiden et al (2011). The purpose of this paper is to find explicit expressions for the radiating waves in the case when the symmetry is only weakly broken. Our strategy is to expand the coefficients in (1) about the symmetric case $c=\alpha=\beta=1$ using a perturbation parameter $\mu \ll 1$. This yields a set of linear equations at $O(\mu)$ described in section 2. These equations are in effect a form of linearisation about the solitary wave (3). They can be decoupled into two equations, one describing the localised perturbation of the solitary wave, developed in section 2.1 and capable of an explicit solution, and the other describing the radiating solitary waves, developed in section 2.2. This latter system is fourth order, and here we exploit the solitary wave amplitude parameter in (3) to obtain approximate solutions in the limit when this amplitude is small. These radiating solitary wave solutions of the $\mathrm{cRB}$ equations are then compared with some relevant numerical simulations in section 3 . We conclude in section 4 .

\section{Asymptotic perturbation from the symmetric case}

It is now convenient to put the cRB equations (1) in the form,

$$
\begin{aligned}
f_{t t}-f_{x x} & =\frac{1}{2}\left(f^{2}\right)_{x x}+f_{t t x x}-\delta(f-g), \\
g_{t t}-g_{x x} & =\frac{1}{2}\left(g^{2}\right)_{x x}+g_{t t x x}+\gamma(f-g)+\mu\left[A g+\frac{1}{2} B g^{2}+C g_{t t}\right]_{x x},
\end{aligned}
$$

where $\left(c^{2}-1\right)=A \mu,(\alpha-1)=B \mu,(\beta-1)=C \mu$, where $A, B, C=O(1)$ and $|\mu| \ll 1$ is a small parameter. Seeking travelling wave solutions of the form $f=f(\xi), g=g(\xi)$ for $\xi=x-v t$, we have 
from $(5)$

$$
\begin{aligned}
\left(v^{2}-1\right) f_{\xi \xi} & =\frac{1}{2}\left(f^{2}\right)_{\xi \xi}+v^{2} f_{\xi \xi \xi \xi}-\delta(f-g), \\
\left(v^{2}-1\right) g_{\xi \xi} & =\frac{1}{2}\left(g^{2}\right)_{\xi \xi}+v^{2} g_{\xi \xi \xi \xi}+\gamma(f-g)+\mu\left[A g+\frac{1}{2} B g^{2}+v^{2} C g_{\xi \xi}\right]_{\xi \xi} .
\end{aligned}
$$

We look for a solution of (6) in the form of the following asymptotic expansions:

$$
f=f_{0}+\mu f_{1}+O\left(\mu^{2}\right), \quad g=g_{0}+\mu g_{1}+O\left(\mu^{2}\right), \quad v^{2}=v_{0}^{2}\left(1+\mu v_{1}+O\left(\mu^{2}\right)\right) .
$$

Substituting (7) into (6), at the leading order we recover the particular solution (3) of the single regularised Boussinesq equation, as discussed above in section 1,

$$
f_{0}=g_{0}=a_{0} \operatorname{sech}^{2}(\varepsilon \xi / 2), \quad \text { where } \quad \varepsilon=\frac{\sqrt{v_{0}^{2}-1}}{v_{0}}, \quad v_{0}^{2}=\frac{1}{1-\varepsilon^{2}}, \quad a_{0}=3\left(v_{0}^{2}-1\right)=\frac{3 \varepsilon^{2}}{1-\varepsilon^{2}} .
$$

At the next order $O(\mu)$ we find that,

$$
\begin{aligned}
\left(v_{0}^{2}-1\right) f_{1 \xi \xi}= & \left(f_{0} f_{1}\right)_{\xi \xi}+v_{0}^{2} f_{1 \xi \xi \xi \xi}-\delta\left(f_{1}-g_{1}\right)+v_{0}^{2} v_{1}\left(f_{0 \xi \xi}-f_{0}\right)_{\xi \xi}, \\
\left(v_{0}^{2}-1\right) g_{1 \xi \xi}= & \left(f_{0} g_{1}\right)_{\xi \xi}+v_{0}^{2} g_{1 \xi \xi \xi \xi}+\gamma\left(f_{1}-g_{1}\right)+v_{0}^{2} v_{1}\left(f_{0 \xi \xi}-f_{0}\right)_{\xi \xi}+ \\
& {\left[A f_{0}+\frac{1}{2} B f_{0}^{2}+v_{0}^{2} C f_{0 \xi \xi}\right]_{\xi \xi} . }
\end{aligned}
$$

Introducing the variables $\phi=f_{1}-g_{1}$ and $\psi=f_{1}+(\delta / \gamma) g_{1}$, the system (9) becomes

$$
\begin{aligned}
\left(v_{0}^{2}-1\right) \phi_{\xi \xi} & =\left(f_{0} \phi\right)_{\xi \xi}+v_{0}^{2} \phi_{\xi \xi \xi \xi}-(\delta+\gamma) \phi-P\left(f_{0}\right)_{\xi \xi}, \\
\left(v_{0}^{2}-1\right) \psi_{\xi \xi} & =\left(f_{0} \psi\right)_{\xi \xi}+v_{0}^{2} \psi_{\xi \xi \xi \xi}+\frac{\delta}{\gamma} P\left(f_{0}\right)_{\xi \xi}+v_{0}^{2} v_{1}\left(1+\frac{\delta}{\gamma}\right)\left(f_{0 \xi \xi}-f_{0}\right)_{\xi \xi}, \\
\text { where } P\left(f_{0}\right) & =A f_{0}+\frac{1}{2} B f_{0}^{2}+v_{0}^{2} C f_{0 \xi \xi}=\left[A+\left(v_{0}^{2}-1\right) C\right] f_{0}+\frac{1}{2}(B-C) f_{0}^{2} .
\end{aligned}
$$

The system is uncoupled and so equations (10), (11) can respectively each be solved for $\phi, \psi$ alone.

\subsection{The localised part of the solution}

First we consider the equation (11) for $\psi$. Introducing a change of variable and integrating twice yields

$$
\psi_{\chi \chi}+4\left(3 \operatorname{sech}^{2} \chi-1\right) \psi=F(\chi)+C_{1} \chi+C_{2}, \quad \chi=\frac{\varepsilon}{2} \xi,
$$

where $C_{1,2}$ are integration constants, and

$$
F=-\frac{4 \delta}{\gamma\left(v_{0}^{2}-1\right)} P\left(f_{0}\right)+\frac{2 v_{1}\left(1+\frac{\delta}{\gamma}\right)}{v_{0}^{2}-1}\left[2 f_{0}+f_{0}^{2}\right] .
$$

We set the constants of integration $C_{1}, C_{2}=0$ since we seek solutions for $\psi$ which are localised as $\chi \rightarrow \pm \infty$. This second order inhomogeneous ordinary differential equation can be solved using the method of variation of parameters. The homogeneous part of (13) is

$$
\psi_{\chi \chi}+4\left(3 \operatorname{sech}^{2} \chi-1\right) \psi=0
$$


and has a localised solution of the form

$$
\psi_{1}(\chi)=\operatorname{sech}^{2}(\chi) \tanh (\chi)
$$

which is proportional to $f_{0 \chi}$, since $(14)$ is the linearisation of the solitary wave equation for $f_{0}$. A second linearly independent solution $\psi_{2}$ of (14) can be found using the Wronskian

$$
\psi_{1} \psi_{2 \chi}-\psi_{2} \psi_{1 \chi}=W
$$

where $W$ is a constant. Solving (16) for $\psi_{2}$ yields

$$
\psi_{2}(\chi)=\frac{W}{32}[60 \chi-32 \operatorname{coth}(\chi)+16 \sinh (2 \chi)+\sinh (4 \chi)] \operatorname{sech}^{2}(\chi) \tanh (\chi) .
$$

Note that $\psi_{2}$ is unbounded as $\chi \rightarrow \pm \infty$. Then using the method of variation of parameters, the general solution of (13) can be written in the form

$$
\psi=\alpha_{1} \psi_{1}+\alpha_{2} \psi_{2}-\frac{\psi_{1}(\chi)}{W} \int_{0}^{\chi} F(\hat{\chi}) \psi_{2}(\hat{\chi}) d \hat{\chi}+\frac{\psi_{2}(\chi)}{W} \int_{0}^{\chi} F(\hat{\chi}) \psi_{1}(\hat{\chi}) d \hat{\chi}
$$

where $\alpha_{1,2}$ are arbitrary constants. Since $\psi_{1} \sim \exp (\mp 2 \chi)$ and $\psi_{2} \sim \exp ( \pm 2 \chi)$ as $\chi \rightarrow \pm \infty$, we must set $\alpha_{2}=0$. Also the first term in the particular solution will contain a secular term proportional to $\chi \exp (\mp 2 \chi)$. This term originates from the term in $F$ proportional to $f_{0} \sim \exp (\mp 2 \chi)$ but can be removed by the choice of $v_{1}$, the first-order speed correction, namely

$$
v_{1}=\frac{\delta}{\gamma+\delta}\left[A+\left(v_{0}^{2}-1\right) C\right] .
$$

Hence we can derive a nonsecular bounded solution of (13):

$$
\psi(\chi)=\alpha_{1} \operatorname{sech}^{2}(\chi) \tanh (\chi)+\frac{3 \delta\left(v_{0}^{2}-1\right)}{\gamma}\left(A-B+v_{0}^{2} C\right) \operatorname{sech}^{2}(\chi)[1-\tanh (\chi)] .
$$

Importantly this solution is localised in $\chi$, and further by choosing $\alpha_{1}=3 \delta\left(v_{0}^{2}-1\right)\left(A-B+v_{0}^{2} C\right) / \gamma$ is symmetric in $\chi$. Indeed then it is proportional to $f_{0}$ and so by itself simply just determines a first-order correction to the solitary wave amplitude.

\subsection{The radiating part of the solution}

The equation for $\phi$ is more difficult to solve since it is a fourth order ordinary differential eqaution and cannot be reduced to second order by integration. Let us first write (10) in the form

$$
L(\phi) \equiv \phi_{\xi \xi \xi \xi}+\left(3 \varepsilon^{2} \operatorname{sech}^{2}(\varepsilon \xi / 2) \phi\right)_{\xi \xi}-\varepsilon^{2} \phi_{\xi \xi}-k^{4} \phi=Q_{\xi \xi}, \quad Q=\frac{P\left(f_{0}\right)}{v_{0}^{2}}
$$

where we recall that $\varepsilon=\sqrt{v_{0}^{2}-1} / v_{0}$ and denote $k^{4}=(\delta+\gamma) / v_{0}^{2}$. The homogeneous form of (21) has four linearly independent solutions, which can be uniquely defined by their behaviour as either $\xi \rightarrow \infty$ or $\xi \rightarrow-\infty$

$$
\begin{gathered}
\phi_{1} \sim \cos m \xi, \quad \phi_{2} \sim \sin m \xi, \quad \phi_{3} \sim \cosh M \xi, \quad \phi_{4} \sim \sinh M \xi, \quad \xi \rightarrow \infty, \\
\hat{\phi}_{1} \sim \cos m \xi, \quad \hat{\phi}_{2} \sim \sin m \xi, \quad \hat{\phi}_{3} \sim \cosh M \xi, \quad \hat{\phi}_{4} \sim \sinh M \xi, \quad \xi \rightarrow-\infty,
\end{gathered}
$$




$$
\text { where } m^{2}, M^{2}=\left(k^{4}+\frac{\varepsilon^{4}}{4}\right)^{1 / 2} \mp \frac{\varepsilon^{2}}{2} .
$$

Importantly, note that as $\varepsilon \rightarrow 0, m, M \rightarrow k$. Next we exploit the symmetry in the operator $L$, that is, if $\phi(\xi)$ is a solution, so too is $\phi(-\xi)$, and so,

$$
\hat{\phi}_{1}(\xi)=\phi_{1}(-\xi), \quad \hat{\phi}_{2}(\xi)=-\phi_{2}(-\xi), \quad \hat{\phi}_{3}(\xi)=\phi_{3}(-\xi), \quad \hat{\phi}_{4}(\xi)=-\phi_{4}(-\xi) .
$$

Also, it is useful to note the solutions

$$
\phi_{5}=\phi_{3}-\phi_{4} \sim \exp (-M \xi), \quad \xi \rightarrow \infty ; \quad \phi_{6}=\hat{\phi}_{3}+\hat{\phi}_{4} \sim \exp (M \xi), \quad \xi \rightarrow \infty .
$$

We again apply the method of variation of parameters, to which end we express the fourth order equation (21) as a $4 \times 4$ system,

$$
\mathbf{U}_{\xi}=\mathbf{A} \mathbf{U}+\mathbf{F}, \quad \text { where } \quad \mathbf{U}=\left(\phi, \phi_{\xi}, \phi_{\xi \xi}, \phi_{\xi \xi \xi}\right)^{\mathbf{T}}, \quad \mathbf{F}=\left(0,0,0, Q_{\xi \xi}\right)^{\mathbf{T}},
$$

and $\mathbf{A}$ is a $4 \times 4$ matrix with rows $(0,1,0,0),(0,0,1,0),(0,0,0,1),\left(\left(k^{4}-S_{\xi \xi}\right),-2 S_{\xi},\left(\varepsilon^{2}-S\right), 0\right)$, where $S=3 \varepsilon^{2} \operatorname{sech}^{2}(\varepsilon \xi / 2)$. Then we seek a solution of $(26)$ in the form

$$
\mathbf{U}=B_{1} \mathbf{U}_{1}+B_{2} \mathbf{U}_{2}+B_{3} \mathbf{U}_{3}+B_{4} \mathbf{U}_{4}=\mathbf{G B}, \quad \mathbf{B}=\left(B_{1}, B_{2}, B_{3}, B_{4}\right)^{\mathbf{T}},
$$

where the vectors $\mathbf{U}_{i}$ for $i=1,2,3,4$ are solutions of the homogeneous part of equation (26) corresponding to $\phi_{i}$, and $\mathbf{G}$ is the fundamental matrix whose columns are $\mathbf{U}_{1}, \mathbf{U}_{2}, \mathbf{U}_{3}, \mathbf{U}_{4}$, that is

$$
\mathbf{G}(\xi)=\left(\begin{array}{cccc}
\phi_{1} & \phi_{2} & \phi_{3} & \phi_{4} \\
\phi_{1 \xi} & \phi_{2 \xi} & \phi_{3 \xi} & \phi_{4 \xi} \\
\phi_{1 \xi \xi} & \phi_{2 \xi \xi} & \phi_{3 \xi \xi} & \phi_{4 \xi \xi} \\
\phi_{1 \xi \xi \xi} & \phi_{2 \xi \xi \xi} & \phi_{3 \xi \xi \xi} & \phi_{4 \xi \xi \xi}
\end{array}\right)
$$

Substituting (27) into (26) yields

$$
\mathbf{B}_{\xi}=\mathbf{G}^{-1} \mathbf{F}
$$

since $\mathbf{G}$ (more specifically each column in $\mathbf{G}$ ) satisfies the homogeneous equation. Integrating (29) yields the general solution of (26):

$$
\mathbf{U}=\mathbf{G}(\xi)\left\{\mathbf{C}+\int_{0}^{\xi} \mathbf{G}^{-1}(\eta) \mathbf{F}(\eta) d \eta\right\}
$$

where $\mathbf{C}=\left(C_{1}, C_{2}, C_{3}, C_{4}\right)^{\mathbf{T}}$ is an arbitrary constant vector. In the sequel, two of these arbitrary constants are chosen to ensure $\phi$ is bounded as $\xi \rightarrow \pm \infty$; that is, they are chosen to remove exponentially growing terms at infinity. The remaining two constants are associated with oscillatory terms at infinity, and these will be chosen to ensure that $\phi \rightarrow 0$ as $\xi \rightarrow \infty$. This is because here the group velocity $v_{g}=p+k p_{k}<p$, since $p_{k}<0$, see Figure 3(a), and so radiated waves will appear only in the region $\xi<0$, see the numerical solution in Figure 2 and our numerical results in section 3. Further progress now requires evaluation of $\mathbf{G}$, that is of $\phi_{i}, i=1,2,3,4$ for all $\xi$. To achieve this we exploit $\varepsilon \ll 1$ in two different ways. 


\subsubsection{Approximation of the variable coefficient term}

Although the fundamental matrix $\mathbf{G}$ is known when $\xi \rightarrow \pm \infty$, some specific knowledge is needed around $\xi=0$ in order to evaluate the integral term in (30). The difficulty in finding exact solutions of the homogeneous equation $L(\phi)=0$ is the variable coefficient term $3 \varepsilon^{2} \operatorname{sech}^{2}(\varepsilon \xi / 2)$ on the left-hand side of (21). In order to make further analytical progress we make the ad hoc approximation that this offending term is removed. Formally we let $\varepsilon \rightarrow 0$, but only in this variable coefficient term. This yields approximate solutions of the homogeneous equation in the form

$$
\phi_{1} \approx \cos (m \xi), \quad \phi_{2} \approx \sin (m \xi), \quad \phi_{3} \approx \cosh (M \xi), \quad \phi_{4} \approx \sinh (M \xi) .
$$

This approximation maintains the correct behaviour of $\phi$ as $\xi \rightarrow \pm \infty$, since in the sequel we seek the solution for $\phi$ only in this far spatial region. Further, with the variable coefficient term neglected in (21), the matrix $\mathbf{A}$ in equation (26) becomes a constant coefficient matrix. Thus, if $\mathbf{U}(\xi)$ is a solution of the homogeneous equation, so is $\mathbf{U}\left(\xi+\xi_{0}\right)$ for any constant $\xi_{0}$. Let $\mathbf{K}(\xi, \eta)=\mathbf{G}(\xi) \mathbf{G}^{-1}(\eta)$ which is the unique matrix solution of the homogeneous equation, such that $\mathbf{K}(\eta, \eta)=\mathbf{I}$ where $\mathbf{I}$ is the unit matrix. It then follows that we have $\mathbf{K}(\xi, \eta)=\mathbf{E}(\hat{\xi}), \hat{\xi}=\xi-\eta$, and $\mathbf{E}(\hat{\xi})$ is the unique matrix solution of the homogeneous equation, such that $\mathbf{E}(0)=\mathbf{I}$. One can write the unique matrix solution in the form

$$
\mathbf{E}(\hat{\xi})=\frac{1}{m^{2}+M^{2}}\left(\boldsymbol{\Phi}_{1}, \boldsymbol{\Phi}_{2}, \boldsymbol{\Phi}_{3}, \boldsymbol{\Phi}_{4}\right),
$$

where the column vectors $\mathbf{\Phi}_{i}=\left(\phi_{i}, \phi_{i \xi}, \phi_{i \xi \xi}, \phi_{i \xi \xi \xi}\right)^{\mathbf{T}}$, for $i=1,2,3,4$, are generated from the approximate solutions (31). The first element of each vector is given by

$$
\begin{array}{ll}
\Phi_{1}=M^{2} \cos (m \hat{\xi})+m^{2} \cosh (M \hat{\xi}), & \Phi_{2}=\frac{1}{m M}\left[M^{3} \sin (m \hat{\xi})+m^{3} \sinh (M \hat{\xi})\right], \\
\Phi_{3}=-\cos (m \hat{\xi})+\cosh (M \hat{\xi}), & \Phi_{4}=\frac{1}{m M}[-M \sin (m \hat{\xi})+m \sinh (M \hat{\xi})] .
\end{array}
$$

In this approximation, it then follows from (30) that the general solution of (26) is

$$
\mathbf{U}=\mathbf{G}(\xi) \mathbf{C}+\int_{0}^{\xi} \mathbf{E}(\hat{\xi}) \mathbf{F}(\eta) d \eta=\mathbf{G}(\xi) \mathbf{C}+\frac{1}{m^{2}+M^{2}} \int_{0}^{\xi} \mathbf{\Phi}_{\mathbf{4}}(\hat{\xi}) Q_{\eta \eta} d \eta
$$

Taking the first element from (33), yields an approximate solution of (10),

$$
\begin{aligned}
\phi & =C_{1} \cos (m \xi)+C_{2} \sin (m \xi)+C_{3} \cosh (M \xi)+C_{4} \sinh (M \xi) \\
& +\frac{1}{m^{2}+M^{2}} \int_{0}^{\xi} \Phi_{4}(\hat{\xi}) Q_{\eta \eta}(\eta) d \eta .
\end{aligned}
$$

Next the integral term in (34) can be integrated twice by parts, and noting that $Q$ is a symmetric function of $\eta$, we can write (34) in the alternative form

$$
\begin{aligned}
\phi= & \tilde{C}_{1} \cos (m \xi)+C_{2} \sin (m \xi)+\tilde{C}_{3} \cosh (M \xi)+C_{4} \sinh (M \xi) \\
& +\frac{1}{m^{2}+M^{2}} \int_{0}^{\xi}[m \sin (m \hat{\xi})+M \sinh (M \hat{\xi})] Q(\eta) d \eta,
\end{aligned}
$$

where $\tilde{C}_{1}=C_{1}+Q(0) /\left(m^{2}+M^{2}\right)$ and $\tilde{C}_{3}=C_{3}-Q(0) /\left(m^{2}+M^{2}\right)$. Then noting that $Q$ is symmetric in $\eta$, and exponentially small in the limit $\xi \rightarrow \pm \infty$, we get that the leading order terms at infinity are given by

$$
\phi \sim\left(\frac{1}{2} \tilde{C}_{3} \pm \frac{1}{2} C_{4}+D\right) \exp ( \pm M \xi), \quad \text { as } \quad \xi \rightarrow \pm \infty
$$




$$
\text { where } \quad D=\frac{M}{2\left(m^{2}+M^{2}\right)} \int_{0}^{\infty} \exp (-M \eta) Q(\eta) d \eta .
$$

Since these terms are secular, we require $\frac{1}{2} \tilde{C}_{3} \pm \frac{1}{2} C_{4}+D=0$, which implies $C_{4}=0, \tilde{C}_{3}=-2 D$. Then finally as $\xi \rightarrow \pm \infty$ we find the oscillatory terms

$$
\phi \sim\left(\tilde{C}_{1}+E_{3}\right) \cos (m \xi)+\left(C_{2} \pm E_{4}\right) \sin (m \xi) \quad \text { as } \quad \xi \rightarrow \pm \infty,
$$

where $\quad E_{3}=-\frac{m}{m^{2}+M^{2}} \int_{0}^{\infty} \sin (m \eta) Q(\eta) d \eta, \quad E_{4}=\frac{m}{m^{2}+M^{2}} \int_{0}^{\infty} \cos (m \eta) Q(\eta) d \eta$.

Finally, we impose an asymmetry condition on $\phi$, namely that we have one-sided radiating solutions in the region $\xi<0$ only. This implies from (37) that $\tilde{C}_{1}=-E_{3}$ and $C_{2}=-E_{4}$, and so

$$
\begin{aligned}
\phi & \sim 0 & \text { as } & \xi \rightarrow+\infty \\
\phi & \sim F_{\text {rad } 1} \sin (m \xi) & \text { as } & \xi \rightarrow-\infty .
\end{aligned}
$$

This one-sided radiating asymptotic solution for $\phi$ depends only on the original parameters in the cRB problem (5) without the presence of any arbitrary constants. From (39) the amplitude of oscillations depends on all three perturbations of $c^{2}, \alpha$ and $\beta$ from 1 and is exponentially small as $\varepsilon \rightarrow 0$.

\subsubsection{Asymptotic solution}

We next consider an alternative approach to only neglecting the variable coefficient term in the governing equation for $\phi$ by systematically exploiting the small parameter $|\varepsilon| \ll 1$. We first express the equation (21) in the form

$$
\begin{aligned}
\phi^{\prime \prime \prime \prime}-k^{4} \phi= & \varepsilon^{2} \phi^{\prime \prime}\left(1-3 \operatorname{sech}^{2} \chi\right)+6 \varepsilon^{3} \phi^{\prime} \operatorname{sech}^{2} \chi \tanh \chi+ \\
& \frac{3 \varepsilon^{4}}{2}(A-\phi) \operatorname{sech}^{2} \chi\left(2-3 \operatorname{sech}^{2} \chi\right)+ \\
& \frac{3 v_{0}^{2} \varepsilon^{6}}{2} \operatorname{sech}^{2} \chi\left[2 C+15 C \operatorname{sech}^{2} \chi\left(\operatorname{sech}^{2} \chi-1\right)+3 B \operatorname{sech}^{2} \chi\left(4-5 \operatorname{sech}^{2} \chi\right)\right],
\end{aligned}
$$

where $\chi=\frac{\varepsilon}{2} \xi$. For $\varepsilon=0$ the solution of (40) will exhibit simple harmonic motion with some frequency $\omega$. Hence we use the Lindstedt-Poincare method by introducing a scaled variable $\tau=\omega \xi$ and seeking an asymptotic solution for $\phi(\tau)$, while also expanding the frequency $\omega$. Under this change of variable in $\xi$ equation (40) becomes

$$
\begin{aligned}
\omega^{4} \phi(\tau)^{\prime \prime \prime \prime}-k^{4} \phi(\tau)= & \varepsilon^{2} \omega^{2} \phi^{\prime \prime}(\tau)\left(1-3 \operatorname{sech}^{2} \chi\right)+6 \varepsilon^{3} \omega \phi^{\prime}(\tau) \operatorname{sech}^{2} \chi \tanh \chi+ \\
& \frac{3 \varepsilon^{4}}{2}(A-\phi(\tau)) \operatorname{sech}^{2} \chi\left(2-3 \operatorname{sech}^{2} \chi\right)+ \\
& \frac{3 v_{0}^{2} \varepsilon^{6}}{2} \operatorname{sech}^{2} \chi\left[2 C+15 C \operatorname{sech}^{2} \chi\left(\operatorname{sech}^{2} \chi-1\right)+3 B \operatorname{sech}^{2} \chi\left(4-5 \operatorname{sech}^{2} \chi\right)\right],
\end{aligned}
$$

where $\chi(\tau)=\varepsilon \tau / 2 \omega$. We then seek an asymptotic solution of (41) as follows

$$
\begin{gathered}
\phi(\tau)=\phi_{0}+\varepsilon \phi_{1}+\varepsilon^{2} \phi_{2}+\varepsilon^{3} \phi_{3}+\varepsilon^{4} \phi_{4}+\varepsilon^{5} \phi_{5}+\varepsilon^{6} \phi_{6}+\cdots \\
\omega=1+\varepsilon \omega_{1}+\varepsilon^{2} \omega_{3}+\varepsilon^{3} \omega_{3}+\varepsilon^{4} \omega_{4}+\varepsilon^{5} \omega_{5}+\varepsilon^{6} \omega_{6}+\ldots
\end{gathered}
$$


Substituting (42) and (43) into (41) yields a sequential set of equations for $\phi_{i}$. Since here we are only concerned with the response to the forcing term, which at leading order is $O\left(\epsilon^{4}\right)$, we can set $\phi_{i}=0, i=0,1,2,3$. The first relevant equations appear at and beyond $O\left(\varepsilon^{4}\right)$, where we have

$$
\begin{aligned}
O\left(\varepsilon^{4}\right): \quad \phi_{4}^{\prime \prime \prime \prime}-k^{4} \phi_{4} & =\frac{3 A}{2} \operatorname{sech}^{2} \chi\left(2-3 \operatorname{sech}^{2} \chi\right) \\
O\left(\varepsilon^{5}\right): \quad \phi_{5}^{\prime \prime \prime \prime}-k^{4} \phi_{5} & =-4 \omega_{1} \phi_{4}^{\prime \prime \prime \prime} \\
O\left(\varepsilon^{6}\right): \quad \phi_{6}^{\prime \prime \prime \prime}-k^{4} \phi_{6} & =-\left(4 \omega_{2}+6 \omega_{1}^{2}\right) \phi_{4}^{\prime \prime \prime \prime}+\phi_{4}^{\prime \prime}\left(1-3 \operatorname{sech}^{2} \chi\right)-4 \omega_{1} \phi_{5}^{\prime \prime \prime \prime} \\
& +\frac{3 v_{0}^{2}}{2} \operatorname{sech}^{2} \chi\left[2 C+15 C \operatorname{sech}^{2} \chi\left(\operatorname{sech}^{2} \chi-1\right)+3 B \operatorname{sech}^{2} \chi\left(4-5 \operatorname{sech}^{2} \chi\right)\right] .
\end{aligned}
$$

Thus $\phi_{4}$ constitutes the leading order solution for $\phi$. It transpires that non-zero higher order correction terms appear only at subsequent even powers of $\varepsilon$, after imposing boundedness and radiation conditions on the solution at each stage.

Leading order solution: Again using the method of variation of parameters, the general solution for the leading order term $\phi_{4}$ is

$$
\begin{aligned}
& \phi_{4}=C_{41} \cos (k \tau)+C_{42} \sin (k \tau)+C_{43} \cosh (k \tau)+C_{44} \sinh (k \tau) \\
& +\frac{1}{2 k^{3}}\left\{\cos (k \tau) \int_{0}^{\tau} L_{4}(s) \sin (k s) d s-\sin (k \tau) \int_{0}^{\tau} L_{4}(s) \cos (k s) d s\right. \\
& \left.+\frac{e^{k \tau}}{2} \int_{0}^{\tau} L_{4}(s) e^{-k s} d s-\frac{e^{-k \tau}}{2} \int_{0}^{\tau} L_{4}(s) e^{k s} d s\right\}, L_{4}(\tau)=(3 A / 2) \operatorname{sech}^{2} \chi\left(2-3 \operatorname{sech}^{2} \chi\right) .
\end{aligned}
$$

Noting that $L_{4}$ is localised and symmetric, we take the limit $\tau \rightarrow \pm \infty$, and get that

$$
\phi_{4} \sim\left(\frac{1}{2} C_{43} \pm \frac{1}{2} C_{44}+F_{1}\right) \exp ( \pm k \tau), \quad \text { as } \quad \tau \rightarrow \pm \infty, \quad F_{1}=\frac{1}{4 k^{3}} \int_{0}^{\infty} L_{4}(s) e^{-k s} d s .
$$

Thus for $\phi_{4}$ to be nonsecular we require $C_{43}=-2 F_{1}$ and $C_{44}=0$. We then find that

$$
\begin{gathered}
\phi_{4} \sim\left(C_{41}+F_{3}\right) \cos (k \tau)+\left(C_{42} \pm F_{4}\right) \sin (k \tau) \quad \text { as } \quad \tau \rightarrow \pm \infty, \\
\text { where } \quad F_{3}=\frac{1}{2 k^{3}} \int_{0}^{\infty} L_{4}(s) \sin (k s) d s, \quad F_{4}=-\frac{1}{2 k^{3}} \int_{0}^{\infty} L_{4}(s) \cos (k s) d s,
\end{gathered}
$$

Finally we impose an asymmetric condition on $\phi$, namely that $\phi$ has one-sided oscillations only in the region $\xi<0$. This requires that $C_{41}=-F_{3}$ and $C_{42}=-F_{4}$. This reduces (49) to

$$
\begin{aligned}
& \phi_{4} \sim 0 \quad \text { as } \tau \rightarrow+\infty \text {, } \\
& \phi_{4} \sim F_{\text {rad }} \sin (k \tau) \text { as } \quad \tau \rightarrow-\infty \text {, }
\end{aligned}
$$

where from $(50)$,

$$
F_{\text {rad }}=-2 F_{4}=-\frac{6 A \pi \omega^{4}}{\varepsilon^{4} \sinh (k \omega \pi / \varepsilon)} .
$$

In the limit $\varepsilon \rightarrow 0$ this leading order asymptotic solution for $\phi \sim \varepsilon^{4} \phi_{4}$ coincides with the previous solution (38) found from the alternative approach in section 2.2.1 only when $B=C$. Indeed, in this present approach, any dependence on $B, C$ can only be found at higher order. 
Higher order correction terms: It is clear that the terms on the right-hand side of the higher order equations (44) and (45), which do not tend to zero in the limit $\tau \rightarrow \pm \infty$, will produce secular terms. Using the same approach as for the leading order equation, we find in order to impose an asymmetric condition, some of these secular terms are non-removable by any choice of arbitrary constants alone, where these arbitrary constants arise from the solutions of the homogeneous equations. Instead we need to use the higher order correction terms in the expansion of the frequency $\omega$.

At $O\left(\varepsilon^{5}\right)$ we can easily remove these secular terms by choosing $\omega_{1}=0$, and as a result, (44) reduces to a homogeneous equation in the same form as that for $\phi_{i}, i=0,1,2,3$ which implies that $\phi_{5}=0$. With this choice of $\omega_{1}$, the equation $(46)$ at $O\left(\varepsilon^{6}\right)$, reduces to

$$
\begin{gathered}
\phi_{6}^{\prime \prime \prime \prime}-k^{4} \phi_{6}=-3 \phi_{4}^{\prime \prime} \operatorname{sech}^{2} \chi+P(\tau)+L_{6 B C}(\tau), \quad P(\tau)=\phi_{4}^{\prime \prime}-4 \omega_{2} \phi_{4}^{\prime \prime \prime \prime}, \\
L_{6 B C}(\tau)=\frac{3 v_{0}^{2}}{2} \operatorname{sech}^{2} \chi\left[2 C+15 C \operatorname{sech}^{2} \chi\left(\operatorname{sech}^{2} \chi-1\right)+3 B \operatorname{sech}^{2} \chi\left(4-5 \operatorname{sech}^{2} \chi\right)\right] .
\end{gathered}
$$

Note that $L_{6 B C}$ depends only on $B, C$ while the dependence on $A$ is contained in $P$. The undetermined $\omega_{2}$ occurs only in $P(\tau)$. Here in $P(\tau)$ as $\tau \rightarrow \pm \infty$ there are trigonometric terms proportional to $\cos (k \tau), \sin (k \tau)$ and these will produce secular terms in $\phi_{6}$ unless they are removed. This can be achieved by choosing $\omega_{2}=-1 / 4 k^{2}$ so that $\omega=1-\varepsilon^{2} / 4 k^{2}+\cdots$. Then $k \tau=k \omega \xi=k\left(1-\varepsilon^{2} / 4 k^{2}+\cdots\right) \xi$ agrees with the exact expression $m \xi$ when $m$ is expanded in powers of $\varepsilon^{2}$, see (24).

With $\omega_{1}, \omega_{2}$ now determined, the general solution of (53) can be found and secular terms removed as for the leading order term. We omit the extensive details, and again imposing the asymmetric condition of one-sided oscillations, we find that

$$
\begin{aligned}
\phi_{6} & \sim 0 \quad \text { as } \quad \tau \rightarrow+\infty, \\
\phi_{6} & \sim-2 K_{1} \cos (k \tau)-2 K_{2} \sin (k \tau) \quad \text { as } \tau \rightarrow-\infty .
\end{aligned}
$$

Here $K_{1}, K_{2}$ are constants given by Fourier-type integrals where the integrand is either $P(\tau)$ or $L_{6 B C}(\tau)$. Since $P(\tau)$ is proportional to $A$, while only $L_{6 B C}$ contains $B, C$ which is the focus of interest here, we need to find only those parts of $K_{1}, K_{2}$ which arise from $L_{6 B C}$. It turns out that there is no such dependence in $K_{1}$, while the contribution to $K_{2}$ is

$$
K_{2 B C}=-\frac{1}{2 k^{3}} \int_{0}^{\infty} \cos (k s) L_{6 B C}(s) d s=\frac{3 v_{0}^{2} \pi\left[B\left(\varepsilon^{2}+k^{2}\right)-C k^{2}\right]}{\varepsilon^{6} \sinh \left(\frac{k \omega \pi}{\varepsilon}\right)} .
$$

Thus the asymptotic solution of (41) as $\tau \rightarrow \pm \infty$, up to $O\left(\varepsilon^{6}\right)$, and keeping only the contribution of $K_{2 B C}$ to $K_{2}$ yields

$$
\begin{array}{ccc}
\phi & \sim 0 & \text { as } \xi \rightarrow+\infty, \\
\phi & \sim F_{\text {rad2 } 2 \sin (k \omega \xi)} \quad \text { as } \xi \rightarrow-\infty . \\
\text { where } \quad F_{\text {rad } 2}=-2 \varepsilon^{4} F_{4}-2 \varepsilon^{6} K_{2 B C}=-\frac{6 \pi\left[A \omega^{4}+B v_{0}^{2}\left(\varepsilon^{2}+k^{2}\right)-C v_{0}^{2} k^{2}\right]}{\sinh \left(\frac{k \omega \pi}{\varepsilon}\right)}
\end{array}
$$

Further corrections to the asymptotic solution for $\phi$ and $\omega$ can be obtained by systematically carrying the expansion beyond $O\left(\varepsilon^{6}\right)$. Note that although $\phi$ is $O\left(\varepsilon^{4}\right)$ in the localised central core, the radiating tail amplitude $F_{\text {rad } 2}$ has no such dependence on the power $\varepsilon^{4}$ but is exponentially small as $\varepsilon \rightarrow 0$. Further this amplitude agrees with that obtained by the previous method in section 2.2 .1 as $\varepsilon \rightarrow 0$; see (38), (39) and note that as $\varepsilon \rightarrow 0, m, M \rightarrow k$ and $\omega \rightarrow 1$. 


\section{Numerical simulations of radiating solitary waves}

Returning to the original variables $f$ and $g$, we have the following asymptotic solution of the system of cRB equations (1),

$$
f=A_{0} \operatorname{sech}^{2}(\chi)+\frac{\mu}{\delta+\gamma}(\gamma \psi+\delta \phi)+O\left(\mu^{2}\right), \quad g=A_{0} \operatorname{sech}^{2}(\chi)+\frac{\mu \gamma}{\delta+\gamma}(\psi-\phi)+O\left(\mu^{2}\right) .
$$

Here we use the localised solution (20) for $\psi$, and then either the asymptotic radiating solution for $\phi$ obtained in section 2.2.1, given by (38), or the analogous expression obtained in section 2.2 .2 given by $(57)$.

In this section we compare these theoretically derived radiating solitary wave solutions of the cRB equations (1) with corresponding numerical simulations, using a pseudo-spectral method which is extended from the work in Engelbrecht et al (2011) for a single regularised Boussinesq equation. We let $x \in[-L, L]$, for finite $L$, and discretise the $(x, t)$ domain into a grid with constant spacings $\Delta x$ and $\Delta t$. The solutions $f(x, t)$ and $g(x, t)$ of the cRB equations (1) are approximated by the solutions $f(i \Delta x, j \Delta t)=f_{\text {num }}$ and $g(i \Delta x, j \Delta t)=g_{\text {num }}$ for $i=1,2, \ldots, N$ and $j=0,1, \ldots$, found from the spectral method. Since we use a spectral method, we impose periodic boundary conditions, but in order to simulate the solutions propagating on the infinite line, we choose $L$ to be sufficiently large. To compare the numerical and theoretical solutions we choose the initial conditions in numerical simulations to coincide with the localised part of the theoretical solutions (59). This comprises of the leading order solitary wave solution and the higher order correction terms given by $\psi$, obtained in section 2.1.

\subsection{Simulations compared with the theory in section 2.2.1}

We first consider the radiating solitary wave solutions derived from the first approach, which we denote as $f_{\text {theory }}, g_{\text {theory }}$. Figures 4 and 5 depict a typical comparison of the theoretical and numerical radiating solitary wave solutions propagating in each component $f$ and $g$. In both figures we find the leading order solitary wave solution is indeed significantly improved by the localised higher order correction term $\psi$. However, as one can see from the close up plots of the oscillating tail region, there are significant discrepancies in the amplitude of oscillations, most evident in Figure 4, which corresponds to perturbations in $c$. On the other hand the wavenumber of the oscillations from the theoretical $(m)$ and numerical solution are in good agreement, as well as with predictions found from the dispersion relation (see Table 1). Although the tail region of the radiating solitary waves shown in Figures 4 and 5 are rather small, they are still considerably greater in magnitude than $O\left(\mu^{2}\right)$, which rules out that the discrepancies are due to higher order terms in the expansions for $f$ and $g$, see (7), not being included.

\begin{tabular}{ccc}
\hline & Wavenumber & Wavelength \\
\hline Dispersion Relation & 0.8110 & 7.747 \\
Numerical & $0.81 \pm 0.1$ & $7.75 \pm 0.1$ \\
$k$ & 0.85 & 7.392 \\
$m$ & 0.8147 & 7.712 \\
$k \omega$ & 0.8139 & 7.719 \\
\hline
\end{tabular}

Table 1: Comparison of wavenumbers and wavelengths of the numerical solution (averaged readings), $m, k$ and that predicted from the linear dispersion relation. All parameter values correspond to Figure 4. 

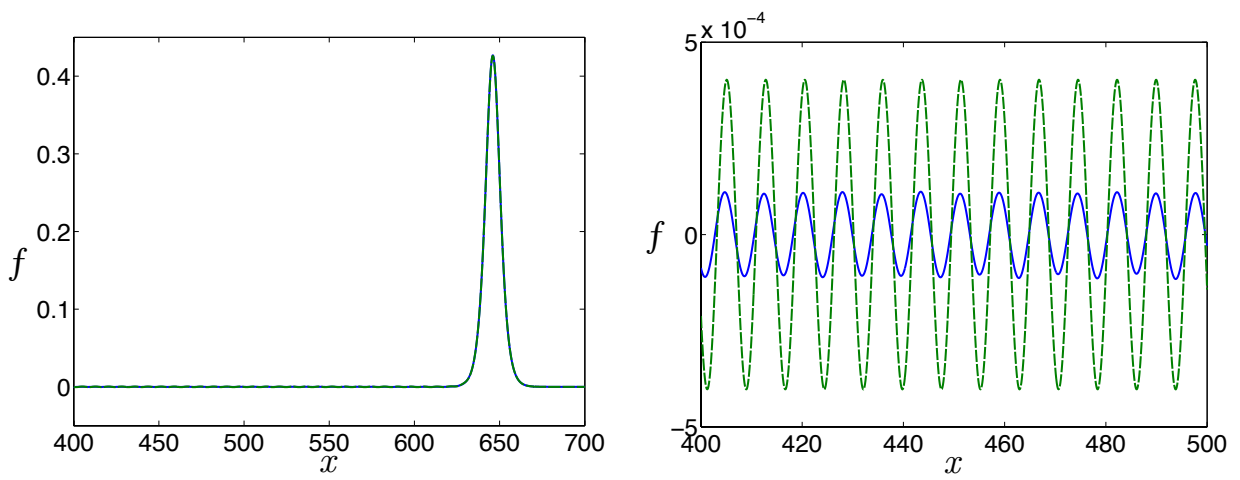

(a) $f_{\text {num }}(-) f_{\text {theory }}(--)$

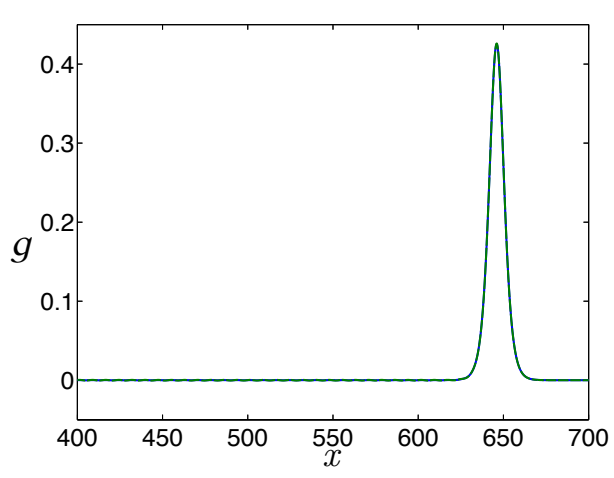

(b) $f_{\text {num }}(-) f_{\text {theory }}(--)$

(c) $g_{\text {num }}(-) g_{\text {theory }}(--)$

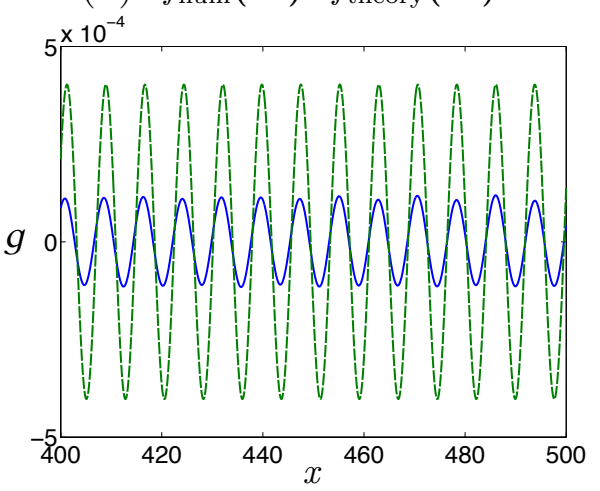

(d) $g_{\text {num }}(-) g_{\text {theory }}(--)$

Figure 4: Numerical solution and theoretical solutions at $t=600$ and a magnification of the oscillating tail. Parameter values: $\varepsilon=0.35, k=0.85, \mu=0.005$ and $A=7, B=C=0$, which implies $m=0.8147, M=0.8868$, $\omega=0.9576, c=1.017, \alpha=\beta=1, \gamma=\delta=0.297, v=1.077, v_{0}=1.068$. Numerical parameters: $\Delta t=0.01$, $L=2000, N=4 \times 10^{5}$. 


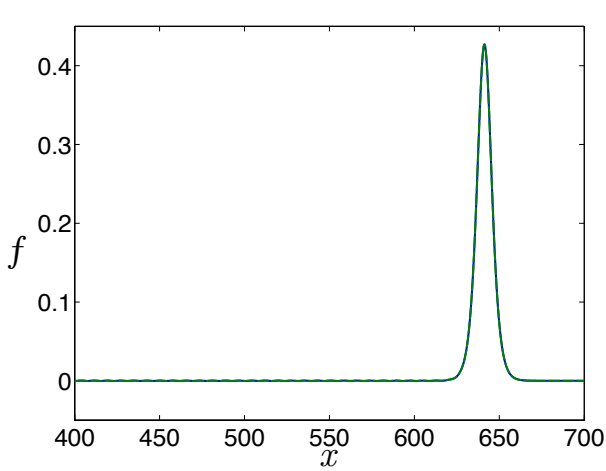

(a) $f_{\text {num }}(-) f_{\text {theory }}(--)$

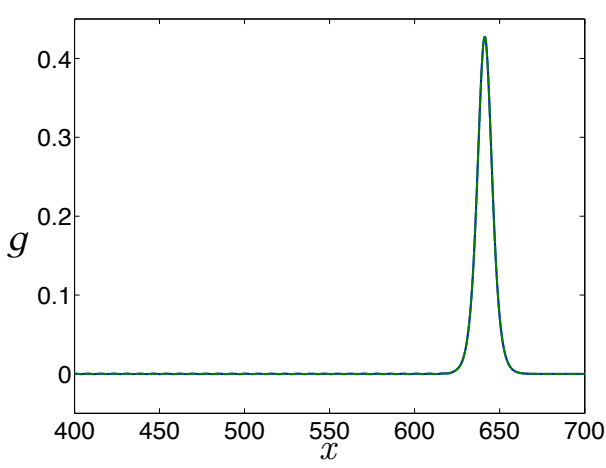

(c) $g_{\text {num }}(-) g_{\text {theory }}(--)$

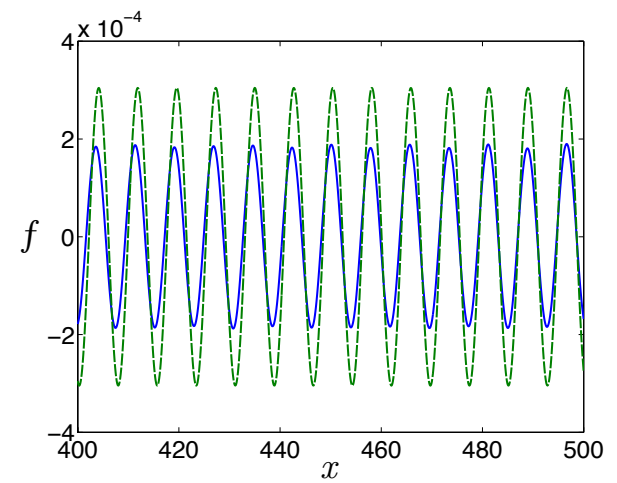

(b) $f_{\text {num }}(-) f_{\text {theory }}(--)$

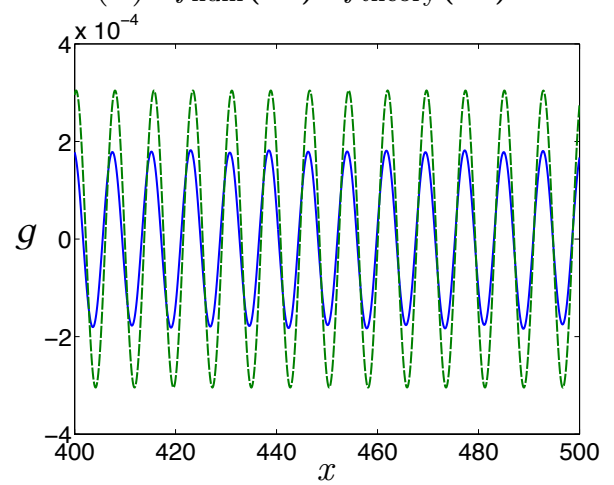

(d) $g_{\text {num }}(-) g_{\text {theory }}(--)$

Figure 5: Numerical solution and theoretical solution at $t=600$ and a magnification of the oscillating tail. Parameter values: $\varepsilon=0.35, k=0.85, \mu=0.005$ and $C=7, A=B=0$, which implies $m=0.8147, M=0.8868$, $\omega=0.9576, c=\alpha=1, \beta=1.035, \gamma=\delta=0.297, v=1.069, v_{0}=1.068$. Numerical parameters: $\Delta t=0.01$, $L=2000, N=4 \times 10^{5}$. 


\subsection{Simulations compared with the theory in section 2.2.2}

Next we consider the alternative asymptotic approach for finding $\phi$ where the outcome is given by (57). Figure 6 depicts typical numerical simulations and the theoretical solution for the same perturbation in the parameter $c$ as in the simulations illustrated in Figure 4, which is reincluded in Figure 6 for comparison purposes. We denote the asymptotic solutions $f$ and $g$ obtained from this perturbation solution for $\phi$ as $f_{\text {pert }}^{i}$ and $g_{\text {pert }}^{i}$, where $\phi$ is taken up to $O\left(\varepsilon^{i}\right)$, for $i=4,6$.

One can see that the solutions for $f$ and $g$ with the higher order terms for $\phi$ included indeed correct the leading order approximations $f_{\text {pert }}^{1}$ and $g_{\text {pert }}^{1}$. This supports the approach outlined in section 2.2.2 as a valid perturbation method for obtaining the oscillatory part of the radiating solitary wave solutions. Moreover, it is clear that this alternative asymptotic approach for finding the oscillatory part of radiating solitary wave solutions of cRB equations is more effective. The solutions for $f$ and $g$ with $\phi$ taken up to $O\left(\varepsilon^{6}\right)$ significantly improves the discrepancy in the amplitude of oscillations from the numerical solution, compared with the solutions for $f$ and $g$ using $\phi$ from the previous approach in section 2.2.1.

Further, we also see an improvement in the amplitude of oscillations in the solutions $f_{\text {pert }}^{1}$ and $g_{\text {pert }}^{1}$, compared with the solutions using $\phi$ from the previous approach denoted here as $f_{\text {theory }}$ and $g_{\text {theory. }}$ It was previously noted that the leading order perturbation solution in $\phi$ is equivalent to the solution for $\phi$ from the previous approach when $\varepsilon \rightarrow 0$. However this is only for leading order expressions $\omega \approx 1, m, M \approx k$. Since $\omega$ appears explicitly in $F_{\text {rad2 }}(58)$, taking higher order terms in $\phi$ and $\omega$, will improve the amplitude of oscillations even for the leading order solution. This is evident in Figure 6, which displays the solutions $f_{\text {pert }}^{1,2}, g_{\text {pert }}^{1,2}$ with the asymptotic solution $\phi$ found with the first non-zero correction term in $\omega$ (and with $m$ and $M$ taken exactly as in the first approach for finding $\phi$ ), where one can already notice a distinct improvement in the accuracy of the leading order solutions $f_{\text {pert }}^{1}, g_{\text {pert }}^{1}$ from the solutions using the previous approach in finding $\phi$.

The same follows for perturbations in the parameter $\beta$ as depicted in Figure 7. In this case $A=0$, and so the leading order asymptotic solution for $\phi$ appears at $O\left(\varepsilon^{6}\right)$, thus the solutions denoted $f_{\text {pert }}^{2}$, $g_{\text {pert }}^{2}$ correspond to the leading order approximations for $\phi$ and are already relatively close to the numerical solution. 


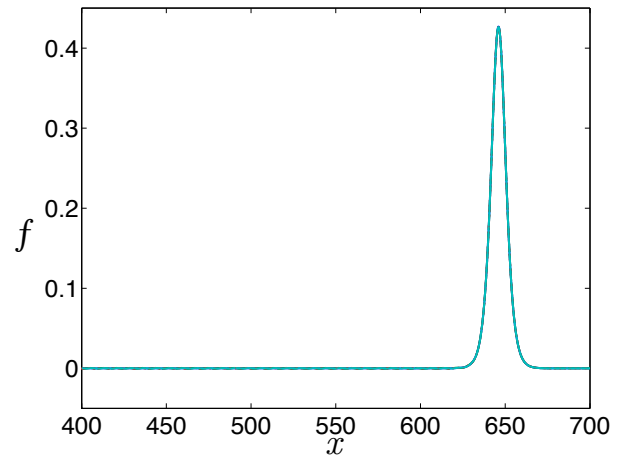

(a) $f_{\text {num }}(-) f_{\text {theory }}(--) f_{\text {pert }}^{1}(\cdots) f_{\text {pert }}^{2}(-)$

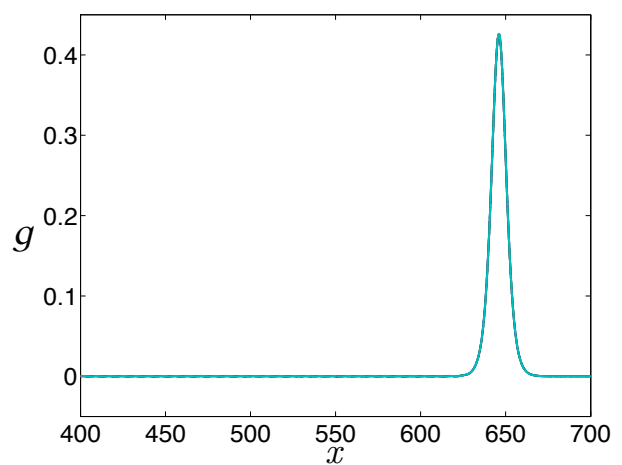

(c) $g_{\text {num }}(-) g_{\text {theory }}(--) g_{\text {pert }}^{1}(\cdots) g_{\text {pert }}^{2}(-)$

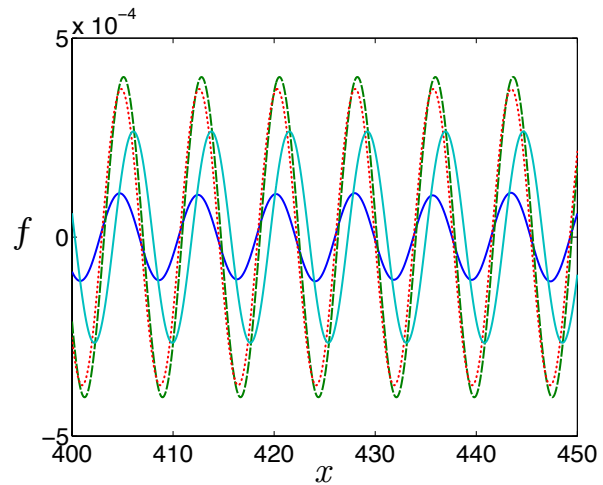

(b) $f_{\text {num }}(-) f_{\text {theory }}(--) f_{\text {pert }}^{1}(\cdots) f_{\text {pert }}^{2}(-)$

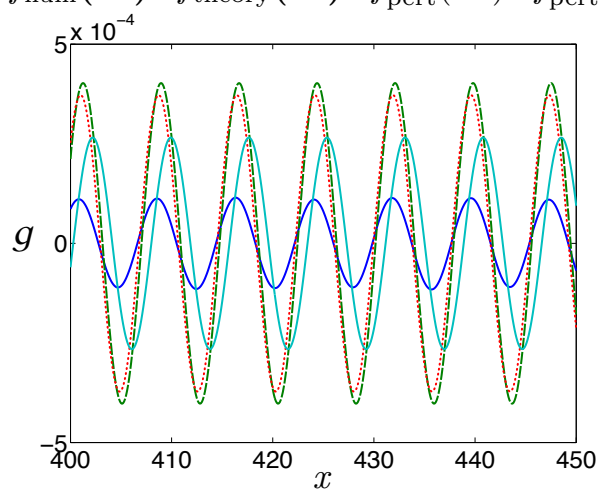

(d) $g_{\text {num }}(-) g_{\text {theory }}(--) g_{\text {pert }}^{1}(\cdots) g_{\text {pert }}^{2}(-)$

Figure 6: Numerical solution and both theoretical solutions at $t=600$. Parameter values: $\varepsilon=0.35, k=0.85$, $\mu=0.005$ and $A=7, B=C=0$, which implies $m=0.8147, M=0.8868, \omega=0.9576, c=1.017, \alpha=\beta=1$, $\gamma=\delta=0.297, v=1.077, v_{0}=1.068$. Numerical parameters: $\Delta t=0.01, L=2000, N=4 \times 10^{5}$. 

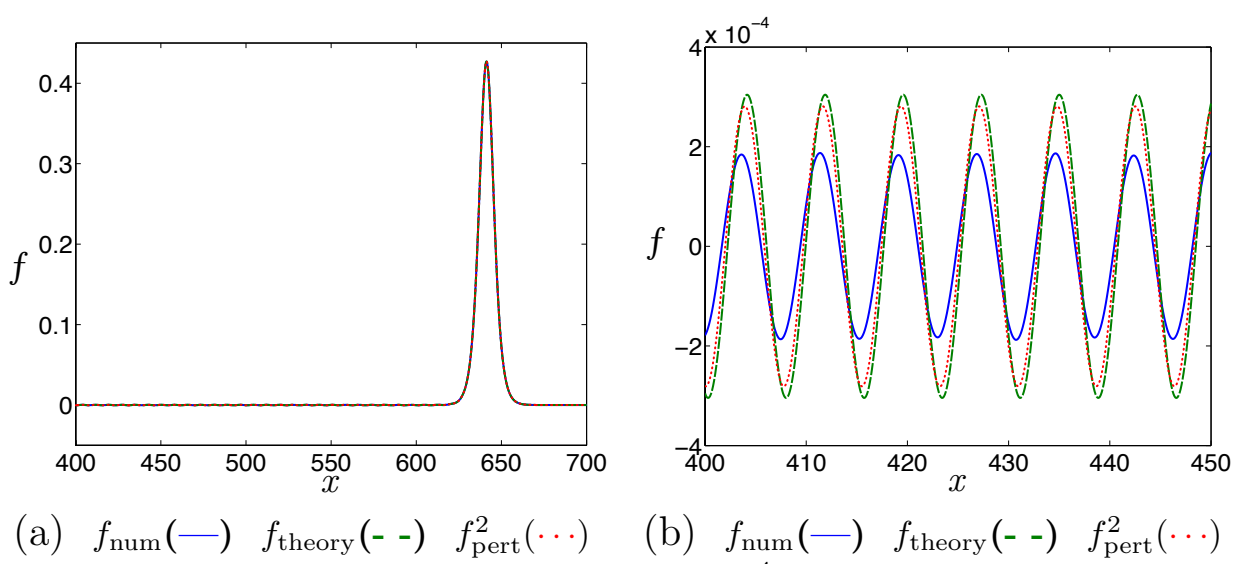

(b) $f_{\text {num }}(-) f_{\text {theory }}(--) f_{\text {pert }}^{2}(\cdots)$
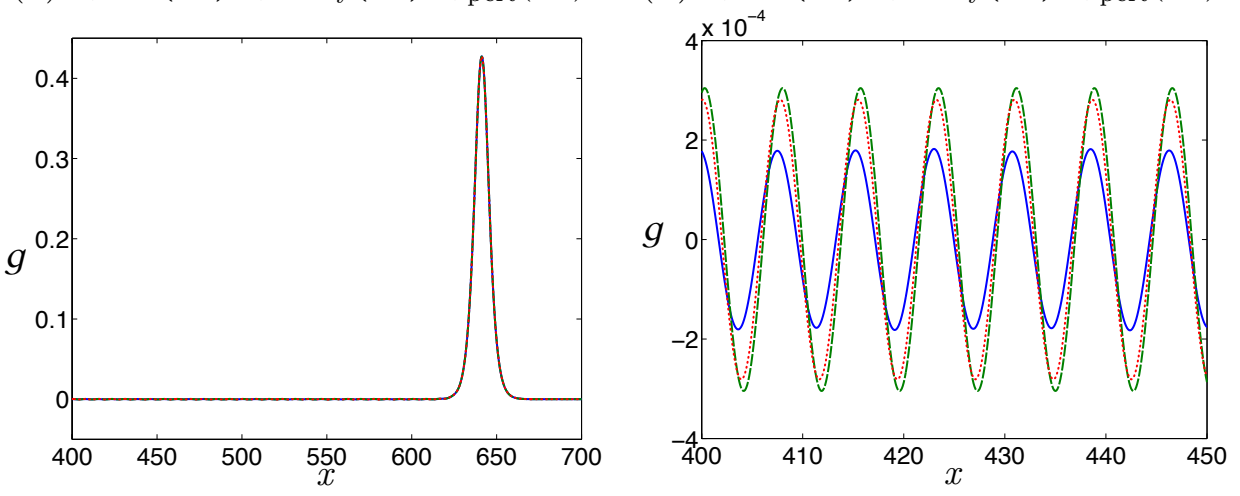

(c) $g_{\text {num }}(-) g_{\text {theory }}(--) g_{\text {pert }}^{2}(\cdots)$

(d) $g_{\text {num }}(-) g_{\text {theory }}(--) g_{\text {pert }}^{2}(\cdots)$

Figure 7: Numerical solution and both theoretical solutions at $t=600$. Parameter values: $\varepsilon=0.35, k=0.85$, $\mu=0.005$ and $A=B=0, C=7$, which implies $m=0.8147, M=0.8868, \omega=0.9576, c=\alpha=1, \beta=1.035$, $\gamma=\delta=0.297, v=1.069, v_{0}=1.068$. Numerical parameters: $\Delta t=0.01, L=2000, N=4 \times 10^{5}$. 


\section{Conclusion}

In this paper we have presented two methods to describe radiating solitary wave solutions inherent in systems of coupled regularised Boussinesq (cRB) equations such as (1). Here we exploited the fact that when the system is symmetric $(c=\alpha=\beta=1$ in (1)) there is an exact solitary wave solution, and then the parameters in the system are slightly perturbed from the symmetric case, represented by a parameter $\mu \ll 1$. This leads to a set of linearized equations (9) which can be solved asymptotically using the method of variation of parameters. The solution represents a radiating solitary wave consisting of a localized central core and one-sided radiation. Our main interest is in the latter, and to find explicit expressions for the amplitude of the oscillations, we employed two different approximation methods, both based on the assumption that the amplitude of the leading order solitary wave is small, represented by $\varepsilon \ll 1$, see (8). In one, described in section 2.2 .1 , we approximated the solutions of the homogeneous linearized equation used in the method of variation of parameters by their far-field expressions, based on the fact that a variable-coefficient term was $O\left(\varepsilon^{2}\right)$. In the other, described in section 2.2.2, we used the Lindstedt-Poincare method in a systematic expansion in powers of $\varepsilon$. In both methods the amplitude of the oscillations was exponentially small with respect to $\varepsilon$, and agreed in the limit $\varepsilon \rightarrow 0$, although small but significant differences emerged for small but finite $\varepsilon$. We note that, although the radiation amplitudes are exponentially small, the present perturbation approach avoids the necessity for the more complicated techniques of exponential asymptotics. However, as pointed out by Akylas and Yang (1995) for instance, it is our ordering that first $\mu \ll 1$ and then $\epsilon \rightarrow 0$ which avoids using exponential asymptotics, and a better procedure would be a simultaneous expansion in $\mu, \epsilon$. Nevertheless, our approach does yield the correct wavenumber for the radiation, and a valid estimate of the amplitude of the radiation which was confirmed by direct numerical simulations, and this is our main purpose here. We also note since the expressions we obtain for the radiation amplitudes depend linearly on three independent parameters $A, B, C$, see (39), (58) for each method respectively, there is a two-parameter family when $\varepsilon \rightarrow 0$ for which the amplitude of the radiating tail is zero. This implies that then there is an embedded solitary wave inside the linear spectrum, see Champneys et al (2001); Yang (2003) for instance.

In section 3 we compared these theoretical results with some typical numerical simulations of the full system (1). The wavenumber of the oscillations was found to be accurately predicted by the theory, but there were discrepancies in the amplitude which was larger in the theory than in the numerical simulations for the cases shown. Importantly we found that the Lindstedt-Poincare method of section 2.2.2 gave better agreement than the ad hoc method of section 2.2.1. In both cases the discrepancies were due to the finite value of $\varepsilon$ and not due to the truncation at $O(\mu)$. Also the second method has the advantage that it can in principle be carried through to higher order in $\varepsilon$.

Recently, the scattering of a long longitudinal radiating bulk strain solitary wave in a delaminated area of a bi-layer with a soft adhesive bonding was modelled numerically by Khusnutdinova and Tranter (2017). The problem was studied within the scope of the system (1), and the results indicated that radiating solitary waves could help us to control the integrity of layered structures with adhesive bondings. The generation of a radiating bulk strain solitary wave in a layered bar with a soft adhesive bonding was experimentally observed by Dreiden et al (2011). The subsequent disappearance of the "tail" in the delaminated area of the bi-layer was observed by Dreiden et al (2012). The estimates for the wavenumber and the amplitude of the "tail" obtained in our paper will help to guide the subsequent numerical and laboratory experiments with adhesively bonded layered structures.

\section{References}

Samsonov, A.M. (2001) Strain solitons in solids and how to construct them. CRC, Boca Raton. 
Porubov, A.V. (2003) Amplification of nonlinear strain waves in solids. World Scientific, Singapore.

Dreiden, G.V., Ostrovsky, Y.I., Samsonov, A.M., Semenova, I.V. and Sokyrinskaya, E.V. (1988) Formation and propagation of deformation solitons in a nonlinearly elastic solid. Zh. Tekh. Fiz., 59, 2040-2047.

Samsonov, A.M., Dreiden, G.V., Porobuv, A.V. and Semenova, I.V. (1998) Longitudinal strain soliton focusing in a narrowing nonlinearly elastic rod. Phys. Rev. B, 57, 5778-5787.

Semenova, I.V., Dreiden, G.V. and Samonov, A.M. (2005) On nonlinear wave dissipation in polymers. Proc. SPIE, Opt. Diagn., 5880, 588006.

Khusnutdinova, K.R. and Samsonov, A.M. (2008) Fission of a longitudinal strain solitary wave in a delaminated bar. Phys. Rev. E, 77, 066603.

Khusnutdinova, K.R., Samsonov, A.M. and Zakharov, A.S. (2009) Nonlinear layered lattice model and generalized solitary waves in layered elastic structures. Phys. Rev. E, 79, 056606.

Dreiden, G.V., Samsonov, A.M., Semenova, I.V. and Khusnutdinova, K.R. (2011) Observation of a radiating bulk strain solitary wave in a solid waveguide. Techn. Phys., 81, 145-149.

Dreiden, G.V., Khusnutdinova, K.R., Samsonov, A.M. and Semenova, I.V. (2012) Bulk strain solitary waves in bonded layered polymeric bars with delamination. J. Appl. Phys., 112, 063516.

Khusnutdinova, K.R. and Moore, K.R. (2011) Initial-value problem for coupled Boussinesq equations and a hierarchy of Ostrovsky equations. Wave Motion, 48, 738-752.

Benilov, E.S., Grimshaw, R. and Kuznetsova, E.P. (1993) The generation of radiating waves in a singularly perturbed Korteweg-de Vries equation. Physica D, 69, 270-278.

Voronovich V.V., Sazonov, I.A. and Shrira, V.I. (2006) On radiating solitons in a model of the internal wave-shear flow resonance. J. Fluid Mech., 568, 273-301.

Bona, J.L., Dougalis, V.A. and Mitsotakis, D.E. (2008) Numerical solution of Boussinesq systems of KdV-KdV type: II. Evolution of radiating solitary waves. Nonlinearity, 21, 2825-2848.

Boyd, J.P. (1998) Weakly Nonlinear Solitary Waves and Beyond-All-Orders Asymptotics. Kluwer, Boston.

Fochesato, C., Dias, F. and Grimshaw, R. (2005) Generalized solitary waves and fronts in coupled Korteweg-de Vries systems. Physica D, 210, 96-117.

Grimshaw, R. and Iooss, G. (2003) Solitary waves of a coupled Korteweg-de Vries system. Math. Comp. Sim., 62, 31-40.

Grimshaw, R. and Joshi, N. (1995) Weakly nonlocal solitary waves in a singularly perturbed Kortewegde Vries equation. SIAM J. Appl. Math., 55, 124-135.

Karpman, V.I. (1993) Radiation by solitons due to higher-order dispersion. Phys. Rev. E, 47, 20732082.

Lombardi, E. (2000) Oscillatory Integrals and Phenomena Beyond all Algebraic Orders. Lecture Notes in Mathematics 1741, Springer, Berlin. 
Vanden-Broeck, J.-M. (1991) Elevation solitary waves with surface tension. Phys. Fluids, A3, 26592663.

Engelbrecht, J., Salupere, A. and Tamm, K. (2011) Waves in microstructured solids and the Boussinesq paradigm. Wave Motion, 48, 717-726.

Akylas, T. R. and Yang, T. -So. (1995) On short-scale oscillatory tails of long-wave disturbances. Stud. Appl. Math., 94, 1-20.

Champneys, A.R., Malomed, B.A., Yang, J. and Kaup, D.J. (2001) Embedded solitons: solitary waves in resonance with the linear spectrum. Physica D, 152-153, 340-354.

Yang, J. (2003) Stable Embedded Solitons. Phys. Rev. Lett., 91, 143903.

Khusnutdinova, K.R. and Tranter, M.R. (2017) On radiating solitary waves in bi-layers with delamination and coupled Ostrovsky equations. Chaos, 27, 013112. 